\title{
La escuela en la sociedad red: Un espacio de encuentro con los valores
}

\author{
Paula Renés-Arellano ${ }^{1}$ \\ https://orcid.org/0000-0003-0932-7694 \\ Universidad de Cantabria, España
}

Schools in the network society: A meeting place with values

Recibido: 08-01-2019

Aceptado: 23-03-2019

\section{Cita Recomendada}

Renés-Arellano, P. (2019). La Escuela en la Sociedad Red: Un Espacio de Encuentro con los Valores. Hamut'ay, 6(1), 112-123.

http://dx.doi.org/10.21503/hamu.v6i1.1578

\section{RESUMEN}

La influencia de los medios de comunicación, de Internet y de las tecnologías en general, es una realidad innegable que requiere de nuevos replanteamientos éticos personales, sociales, políticos, económicos, culturales y educativos, que preserven los auténticos principios democráticos en las sociedades actuales. La Sociedad Red como estructura social de la era de la información es uno de los pilares que fundamentan el trabajo presentado, a través de esta el objetivo es realizar un acercamiento teórico hacia su definición y la relación existente con los efectos de la presencia de Internet y los nuevos medios tecnológicos y audiovisuales que evidencian una realidad social en continua transformación que requiere de agentes educativos comprometidos con la misma. El método aplicado ha sido descriptivo a través de una búsqueda bibliográfica que apoya la reflexión-teórica de la misma. Los resultados se dirigen hacia propuestas de cómo se otorga a la escuela y a la sociedad, la oportunidad de promover los valores sociales y personales albergados con la corresponsabilidad, la justicia con baluartes de los procesos educativos de los más jóvenes. Desde este planteamiento, se avanza hacia la relación existente entre la Sociedad Red y los valores como elementos fraguadores de las sociedades democráticas, finalizando con las conclusiones y propuesta de retos, oportunidades y ventajas que supone educar en valores en las escuelas en la sociedad actual.

Palabras Clave: Valores, Internet, escuela, democracia, alumnos, profesores, tecnologías.

\section{Abstract}

The influence of the media, the Internet and technologies in general, is an undeniable reality that requires new ethical, personal, social, political, economic, cultural and educational rethinking that

1 Doctora y profesora en la Facultad de Educación, adscrita al área de Teoría e Historia de la Educación de la Universidad de Cantabria (España). Las líneas de investigación se vinculan a la educación mediática, TIC y valores. Miembro de la Red Interuniversitaria Euroamericana de Investigación sobre Competencias Mediáticas para la Ciudadanía (ALFAMED). E-mail: paula.renes@unican.es 
preserves the authentic democratic principles in current societies. The Network Society as a social structure of the information age is one of the pillars that underlie the work presented. Through it, the objective is to make a theoretical approach to its definition and the existing relationship with the effects of the presence of the Internet and the new technological and audiovisual media that demonstrate a social reality in continuous transformation that requires educational agents committed to it. The applied method has been descriptive through a bibliographic search that supports the theoretical-reflection of it. The results lead to proposals for how the school and society are given the opportunity to promote the social and personal values hosted by co-responsibility, justice with bastions of the educational processes of the youngest. From this approach, progress is made towards the relationship between the Network Society and the values as fragmenting elements of democratic societies, ending with the conclusions and proposals of challenges, opportunities and advantages that involves educating in values at schools in today's society.

Keywords: Values, Internet, school, democracy, students, teachers, technologies.

\section{INTRODUCCIÓN}

El actual epicentro de la sociedad se enmarca en las Tecnologías de la Información y la Comunicación (TIC), la presencia de Internet y los nuevos modelos de comunicación social y ciudadana, llevando a reconocer la era de la información como una sociedad caracterizada por la inmediatez, la fragilidad o la facilidad del acceso a la información; pero también, una sociedad cargada de valores, retos y proyectos humanizadores. Internet $y$ en concreto, las posibilidades que se ofrecen a través de los móviles o dispositivos inteligentes, las ventanas abiertas al mundo, han llevado a una clara "conectividad total" o, lo que es lo mismo "Internet del todo". Este influjo de los medios de comunicación, de Internet o de las tecnologías en general, es innegable; se considera un proceso dinámico en el que la escuela, como institución socializadora, se ha visto enmarcada. Algunos autores como Castells (2003), ya definieron la sociedad actual como la Sociedad Red: «la nueva estructura social de la Era de la Información, basada en redes de producción, poder y experiencia» ( $\mathrm{p}$. 350). Esta descripción ha sido confirmada, y permanece aún vigente con autores como Medina $\&$ Ballano (2015), quienes también la denominan "sociedad mediatizada", en la que los medios y las tecnologías se convierten en posibles agentes educativos. En esta línea Jiménez (2002) conduce su aportación hacia el concepto de "techné", aquello que permite a la persona designar algo que no es capaz de describirse por cualidades de su propia naturaleza (physis), sino que es considerado elaborado, producido o creado por el individuo (Cabrera, 2004), y, por tanto, es "constitutivo de lo humano” (Fernández \& López, 2018, p.138). Si las tecnologías, los medios de comunicación o Internet son consideradas un producto social y cultural inherente al propio individuo, la educación se convierte en el baluarte necesario para entender, comprender y reflexionar sobre la influencia de las mismas en el desarrollo personal e interpersonal de cada sujeto.

En este contexto, parece necesario plantearse realmente qué papel juega la Sociedad Red en el mundo globalizado y por tanto, cómo se puede conseguir que los ciudadanos se empoderen de las capacidades, destrezas y habilidades necesarias que les permitan ser críticos, activos y comprometidos con las decisiones y acciones que toman en relación con las situaciones en las que se desenvuelven; cómo la escuela, queda más que justificada en la necesidad de experimentar estos avances en sus tareas educativas.

Es asumible, pero a veces, complejo, entender que el acceso a la información es un derecho y una vía fundamental para conocer el mundo e interaccionar con él. Sin embargo, el consumo pasivo de 
esta información no garantiza la adquisición de competencias éticas, críticas y responsables sobre el uso de los medios de comunicación, de Internet o de las tecnologías en general. La "sociedad líquida" (Bauman, 2007) en la que la celeridad marca muchas de las relaciones sociales, no garantiza el aprendizaje de destrezas, valores, conocimientos $y$ actitudes que requieren ser asimilados en este nuevo contexto. Es necesario seguir favoreciendo desde las escuelas procesos educativos que permitan a los niños, ir progresivamente adquiriendo las habilidades necesarias que les conviertan en verdaderos consumidores con miradas críticas ante, para y por lo que ven, escuchan y asimilan.

Investigaciones como las de Ruiz, Bernal, Gil \& Escámez, 2012; Martínez 2015, recogen la necesidad de desarrollar una educación en valores dentro de la escuela. Según Bindé (2004), plantearse y reflexionar sobre los valores, es el resultado de analizar y ser consciente de que las sociedades cambian, se transforman, y ello deriva en determinados efectos en la manera de relacionarnos socialmente ante las tecnologías. En este sentido, una de las responsabilidades sociales y ciudadanas es formar a niños y jóvenes, especialmente si el foco de atención es la escuela, esta como "prodiseñadores de contenido" (Hernández-Serrano, Renés-Arellano, Graham, \& Greenhill, 2017).

Por lo que, el objetivo de este estudio es realizar un acercamiento teórico-reflexivo sobre la Sociedad Red, una realidad en la que Internet y sus efectos, así como los nuevos medios tecnológicos y audiovisuales, evidencian un contexto social en continua transformación que requiere de agentes educativos comprometidos con la misma.

\section{MÉTodo}

Se realizó una búsqueda bibliográfica para el análisis de la revisión de la literatura que apoya la reflexión-teórica de la misma durante el primer trienio de 2019. Se empleó un diseño de tipo descriptivo (Day, 2005), lo cual sirvió para seguir los pasos y procedimientos para la redacción final del estudio, identificando documentos bibliográficos en diversas bases de datos como son; ESCI, WOS y Scopus y otras fuentes como las publicaciones en libros impresos y digitales (Tabla 1). Los descriptores empleados (tanto en castellano como en inglés) fueron: valores, alumnos, profesores, sociedad, Internet, medios de comunicación, democracia, tecnologías y escuela. Se seleccionaron aquellos documentos que informaron de los aspectos formales que debía recoger la revisión y la lectura crítica de los mismos. Los objetivos de esta primera fase fueron: resumir la información sobre el tema, identificar aspectos conocidos o controvertidos, identificar aproximaciones teóricas ya elaboradas sobre la temática, proporcionar información vinculada a esta revisión, reflexionar sobre los temas abordados tomando en consideración la Sociedad Red, los valores y la educación como focos de interés, mostrar evidencias de esta y ofrecer retos, oportunidades y propuestas finales que ayuden a mejorar avanzar hacia la relación existente entre la Sociedad Red y los valores como elementos fraguadores de las sociedades democráticas y en la escuela.

Tabla 1

Bases de datos y artículos seleccionados

\begin{tabular}{lll}
\hline \multicolumn{1}{c}{ Bases de datos } & tipo de documento & $\begin{array}{l}\text { Artículos se- } \\
\text { leccionados }\end{array}$ \\
\hline Wos & & 16 \\
Scopus & & 10 \\
Esci & Revistas científicas & 3 \\
Latindex & & 10 \\
Otras & & 4 \\
Libros y Documen- & Libros editoriales es- & 15 \\
tos Internacionales & pañolas en SPI & \\
& Libros internacionales & 7 \\
& (inglés) & 2 \\
& Otros & \\
\hline
\end{tabular}

Fuente: elaboración propia 2019

La estrategia de búsqueda, una vez seleccionadas las bases de datos, y fijados los descriptores, fue seguir los pasos que plantea Maeda (2006): ordenar, rotular, integrar y priorizar la información recogida para lo que se inició el planteamiento y estructura del trabajo siguiendo los recursos documentales obtenidos y llevando el hilo conductor del objetivo del trabajo iniciando la reflexión teórico-descriptiva desde la Sociedad Red, los valores, la democracia y la escuela. Para ello, en el apartado de conclusiones, que es la segunda fase, 
se indican estos temas.

Un acercamiento a los valores en la escuela desde la educación en la Sociedad Red

A menudo se escucha en prensa y en los medios de comunicación que los nuevos modelos de globalización están acusando las diferencias entre pueblos y culturas, y aumentando la brecha de la fragmentación social; una preocupación que ha sido abordada en diferentes espacios de debate y reflexión como son los organismos internacionales. En la actualidad, existen grandes y diversas tensiones suscitadas por los cambios tecnológicos, así como sociales y económicos, que plantean cómo afrontar e integrar elementos cercanos y lejanos, tradicionales, actuales, particulares y universales, o materiales y espirituales, $y$, por tanto, cómo las personas son capaces de asumirlo y adaptarlo a sus realidades desde una visión personal y grupal (UNESCO, 2015). Estos elementos descritos son los que alimentan las transformaciones actuales a nivel social y marcan los patrones culturales y comunicativos de todos los grupos sociales. En este contexto aparecen también los valores, relacionados con la cultura de sociedad. Es la cultura y los valores, los que permiten, entre otros aspectos, comprender de forma global las capacidades, conocimientos y moralidad de determinados grupos sociales (Arribas \& Torrego, 2008).

La adquisición de valores es un proceso dinámico y permanente que permite asumir las normas sociales, convivir en comunidad, desarrollarse integralmente y respetar a los demás. Actualmente, se habla de una crisis de valores (Buxarrais, 1997); como señala Tornero $(2017$, p.5), "por el simple hecho de habernos trasladado a la red, de vivir de modo virtual, hemos puesto en crisis algunos de los valores que imperan en nuestro día a día”. Es el resultado de las sociedades globales actuales, caracterizadas por su heterogeneidad, y cada vez más acusadas por los nuevos registros comunicativos que exige la Sociedad Red. En palabras de Mínguez (2014), existe una fractura de criterios y referencias de convivencia social que pueden afectar a las relaciones interpersonales y es resultado del modo de vida de las personas desde comienzos de este siglo. Es por ello, que se requiere una educación reformulada, un pilar fundamental que vele por la búsqueda de la responsabilidad de todos los que formamos parte de la sociedad, en el que la escuela juega un papel primordial (Mayor, 2007).

Este progreso tecnológico en el que los medios de comunicación, Internet o las tecnologías están presentan en nuestra cotidianeidad genera el surgimiento de cuestiones éticas que pueden ser complejas de afrontar, pero necesarias (Albizuri, Samaniego \& Torrientes, 2001). La multiculturalidad actual es a su vez, junto con estos elementos, un reto educativo fundamental, porque se presenta como la oportunidad de poder desarrollar una ciudadanía democrática y dialógica, que favorezca el intercambio cultural y la comprensión social. La escuela, en este sentido, se puede convertir en el espacio de reflexión y acción que favorezca el desarrollo de identidades personales, culturales y sociales que permitan participar a las personas bajo principios de libertad (Besalú, 2002).

El cuidado y la preservación de valores universales como la libertad han sido y siguen siendo, objeto de análisis, reflexión y defensa desde diversos organismos, más concretamente y realizando un acercamiento a documentos internacionales, parece necesario detenerse en la Declaración Universal de Derechos Humanos de diciembre de 1948, que ya en su artículo 26 promulgó que toda persona tiene derecho a su pleno desarrollo:

La educación tendrá por objeto el pleno desarrollo de la personalidad humana y el fortalecimiento del respeto a los derechos humanos y a las libertades fundamentales; favorecerá la comprensión, la tolerancia y la amistad entre todas las naciones y todos los grupos étnicos o religiosos; y promoverá el desarrollo de las actividades de las Naciones Unidas para el mantenimiento de la paz (ONU, Asamblea General, Declaración Universal de los Derechos Humanos. Art.26. p.8).

También otras instituciones han dejado patente esa necesidad de luchar no solo por los derechos humanos, sino también por las libertades. Valores y derechos que la educación puede promulgar y que son propios de las sociedades contemporáneas, tal y como reflejan las diferentes Decla- 
raciones de Derechos Humanos, en las que se han reconocido el derecho a la educación como el instrumento base para el pleno desarrollo de la persona. También la UNESCO en 1960 y el Convenio para la protección de los derechos humanos y de las libertades fundamentales, aprobado en Roma el 4 de noviembre de 1950 hacen eco de esa realidad.

Entender la autonomía del sujeto es respetar la autonomía personal, la libertad individual, de creencias, expresión y educación, aspectos que se recogen, en el artículo 27 de la Constitución Española:

1. Todos tienen el derecho a la educación. Se reconoce la libertad de enseñanza.

2. La educación tendrá por objeto el pleno desarrollo de la personalidad humana en el respeto a los principios democráticos de convivencia y a los derechos y libertades fundamentales.

Actualmente, ya se recoge desde organismos internacionales como la UNESCO, el Parlamento Europeo, la Comisión Europea y la ONU a través de su programa de la alianza para las civilizaciones, la importancia y necesidad de una educación en valores actualizada, en la que las personas logren ser críticas y activas ante las realidades sociales, culturales, económicas, tecnológicas o mediáticas.

Parece imprescindible plantearse y reflexionar sobre cómo y de qué manera desde la escuela se cuida y se capacita a los nińos para que sean conscientes de los valores y contravalores que se transmiten en las redes. Según Garitaonandia \& Garmendia (2007) los jóvenes con mayores problemas en Internet lo ocultan a los padres, por lo que la necesidad de conocer, analizar e investigar realmente qué tipo de valores o contravalores adquieren los niños a través de Internet, parece necesaria, porque promueve valores morales e influye en el desarrollo moral (Koh, 2014) y en la construcción de su identidad digital y personal (Zhao, Grasmuck, \& Martin, 2008). Si la Convención de las Naciones Unidas sobre los derechos del niño habla de la necesidad de preservar los datos personales de los niños; la escuela, como institución socializadora, debe ser sensible y estar preparada para afrontar esta realidad a través de intervenciones educativas ajustadas a estas necesi- dades propias de la interconexión, el acceso libre a la información y la celeridad para compartir información.

Los valores de una sociedad: del pensamiento a la acción para comprender las realidades actuales en educación

$\mathrm{Si}$ entre las funciones fundamentales de la escuela prima la educación, es ahora el momento de explicar cómo es entendida: como un proceso intencional que debe favorecer el pleno desarrollo de la persona es uno de los estandartes de las sociedades democráticas, tal y como defendió Bernardini (2010). Porque ofrece el marco ideal para adquirir las habilidades y destrezas necesarias que permiten a cada individuo pensar y tomar decisiones adecuadas y ajustadas a las realidades. La educación, en este sentido, se convierte en un factor determinante para la promoción de valores universales aceptados y respetados por todos, y, en consecuencia, compartidos socialmente.

Desde esta perspectiva, y siguiendo a los autores García Aretio, Ruíz \& García Blanco, (2010), la educación promoverá la adquisición y la asimilación de valores propios en cada individuo, y deberá favorecer el desarrollo de una ciudadanía crítica, participativa y activa. En este sentido, Hernández \& Eyeang (2017) afirman que los valores propios de una sociedad o comunidad son aquellas pautas de actuación individual y social que son aceptadas para la adecuada convivencia y que de forma transgeneracional serán respetadas por todos los miembros de la comunidad o grupo social. Por ende, los valores de una sociedad son aquellos que además de ser adquiridos, necesarios y observados, también son estimados como válidos. En este contexto, la educación cobra sentido, porque es a través de ella como cada persona logra ese proceso de adquisición de valores, los dota de significado y los transfiere al ámbito individual (Martínez, Esteban \& Buxarrais, 2011).

Es por ello, que surge la incertidumbre de cómo esta nueva cultura mediática que está patente actualmente en la Sociedad Red, ejerce efectos en las actuaciones de las personas (Colom \& Melich, 1993). Y cómo pueden influir en la construcción de una moral adecuada, de valores racionales que 
permitan ser integrados en la cotidianeidad personal y social. Esta reflexión requiere de un análisis pormenorizado de estos interrogantes desde una óptica responsable y comprometida de aquellos que ofrecen los medios en el desarrollo de la persona (García-Ruíz, Ramírez, \& Rodríguez, 2014).

El panorama actual de Internet está generando formas diferentes de interaccionar, comunicarnos y dar sentido a las realidades en las que se vive, está generando nuevos hábitos derivados del papel de agentes socioeducativos que han adoptado estas tecnologías. Y es que "somos partícipes y espectadores de una época en donde los nuevos canales de comunicación, especialmente Internet, se han convertido en noticias recurrentes" (Garrido-Cabezas, 2011, p.1376).

Internet, así como los medios de comunicación y las tecnologías están promoviendo nuevos hábitos, y un mundo de valores nuevos, con una moral diferente y adaptada a estas realidades.

El problema surge cuando el anonimato que se mantiene en la Red hace que se pierdan los valores realmente importantes, como son el respeto a otra persona o la responsabilidad de las acciones que tomamos en los procesos comunicativos en Internet. Internet ofrece un gran potencial, tal y como afirman autores como, Agarwal, 2000; Agarwal \& Venkatesh, 2002; Carmel \& Agarwal, 2002; Briñol., et al., 2004; Reig, 2018, quienes estudian la relación de la cognición humana, los valores y el uso de Internet. En concreto, comparten la idea de que existen diferentes variables que influyen, desde perspectivas exógenas, en las actitudes y usos de Internet entre las personas, más específicamente, afirman que las habilidades y las motivaciones están estrechamente relacionadas con la interacción que se establece en la Red en la que la persona es capaz de diversificar la comunicación a través de diferentes canales online y requiriendo por su parte, la puesta en marcha de mecanismos cognitivos y habilidades que le permitan establecer, iniciar o mantener una relación interactiva a través de Internet. A ello hay que sumar, que las personas buscan en las comunicaciones online las finalidades actitudinales, eso significa que necesitan conocer y controlar esos procesos de interrela- ción porque puede ser que esa relación estableci$\mathrm{da}$, coincida con las creencias o valores personales, pero se precisa conocer realmente qué significado cobran esas relaciones y las acciones derivadas de las mismas (Orantes, 2011).

Es en este contexto desde donde se precisa una educación en valores, que prepare y forme integralmente a los niños para su desarrollo y para una adecuada adquisición de valores necesarios en los nuevos entornos mediáticos y tecnologizados. Internet se ha convertido hoy en día en un instrumento fundamental para la construcción de valores socialmente compartidos porque en su utilización diaria los valores afloran constantemente, las decisiones responsables o irresponsables se relacionan con esta, y las conductas éticas suelen estar pautadas por los aprendizajes que se derivan de la interrelación. En concreto, Tornero (2017, p.15) afirma que "en la actualidad existen ciertos vacíos éticos en el mundo digital, lo que puede dar lugar a problemas y malentendidos-que provocan que muchas personas teman usar las TIC". En este sentido, es cierto que el acceso a la información no supone haber adquirido las capacidades, habilidades y actitudes que permitan a la persona, niño, joven o adulto, a ser competente ante el contenido que se muestra en las redes, y menos aún, que se participe de forma crítica y responsable ante el uso de Internet. Es por ello, que desde este trabajo se aboga por realizar procesos de reflexión crítica, responsable y ética sobre la sociedad digital actual (Buxarrais, Martínez, Puig \& Trilla, 1990; Aparici, 2010; Caldeiro \& Aguaded, 2015), que permitan adquirir ciertos valores universalmente compartidos y rechazando aquellos contravalores que, desde la óptica de la Red, no favorecen la construcción de una adecuada moral en los más jóvenes.

Transformaciones sociales y escuela: hacia una ciudadanía democrática en un mundo tecnologizado

La pluralidad de concepciones e ideas que supone la existencia de los cambios de las sociedades hace que las personas se enfrenten diariamente a conflictos de valores y tomen decisiones siguiendo sus propios patrones de escala de valores e interpreta- 
ción de las realidades. Ante este planteamiento, se hace necesaria una educación que mire el plano axiológico como un todo indiscutible; una escuela que promueva hábitos de convivencia sustentados en valores democráticos en los que el diálogo, la escucha o la participación de todo el alumnado sea tomado en cuenta (Abad, 2018). Y es que los hábitos personales forman parte de la cultura de una escuela, de su forma de entender las realidades e interacciones humanas $y$, además, se precisa realizar prácticas coherentes que consigan efectos positivos en las propuestas y toma de decisiones diarias (Martínez, Puig \& Trilla, 2003).

La escuela se convierte en el espacio a través del cual se producen procesos de socialización y transmisión de ideologías, promoviendo el desarrollo de la personalidad de cada niño (Deval, 2002; Martínez, Esteban \& Buxarrais, 2011). Es por ello, por lo que normalmente la sociedad confía en que, desde su posición institucional, socializadora y educadora, se fomente la transmisión de valores democráticos, siendo fundamentales los roles que ejercen todos los miembros de la comunidad educativa, en especial el profesorado y las familias, como agentes más cercanos a los niños, ya que ejercen una influencia fundamental en la educación en valores.

No se puede seguir avanzando en el camino de la justificación de los valores en la sociedad tecnologizada actual, sin detenerse en las palabras que pronunciaron Ortega \& Mínguez (2004) sobre la familia y su función como transmisora de valores:

El cambio profundo producido en la sociedad postindustrial sociológicamente laica, incorporada a los avances tecnológicos de la información, ha llevado consigo el debilitamiento de la institución familiar como ámbito autosuficiente y mecanismo básico de transmisión de valores; pero también ha supuesto la recuperación de un nuevo discurso sobre la familia (p. 37).

Es la familia, junto con el profesorado y la escuela quienes deben primar una educación por, para y ante valores promotores de principios democráticos. $\mathrm{Y}$ es que convictos de realismo, lo cierto es que es evidente el inexorable funcionamiento de la ciudadanía hacia un cosmopolitismo que avanza por la tecnociencia, tal y como defiende Maceiras (2015), un camino en el que la familia promueva principios y prácticas educativas coherentes con las realidades tecnológicas y digitales existentes, que busquen el desarrollo de hábitos éticos e intelectuales con la formación de los niños. La identidad personal se fragua en consonancia con la singularidad propia, pero también, en relación con los otros y el contexto en el que se vive, aspectos que ya defendieron en su momento autores de gran relevancia como fueron Hegel y Ortega.

$\mathrm{Si}$ se toman estas afirmaciones como ciertas, la esencia educativa en la escuela y la ética, estará cargada de compromisos y deberes que ha de asumir cada agente educativo y alumnado, en su acción diaria. Porque la construcción de la moral, el desarrollo de principios éticos y la asunción de valores democráticos en el proceso educativo de los niños, favorecerá la adquisición de hábitos, acciones y conductas responsables y éticamente correctas. En este sentido y siguiendo a Maceiras (2015), se comparten los valores, que aún discutibles por la permeabilidad e intangibilidad de las sociedades digitalizadas en continua transformación, se hacen necesarios para solventar esa crisis de valores sobre la que se reflexiona habitualmente. Se precisan referencias axiológicas seguras que permitan a niños y jóvenes construir sus propios juicios morales desde pilares sólidos y coherentes con los retos que surjan en las sociedades. Es por ello, que a pesar de la complejidad de las sociedades y de los valores que en ellas se transmiten, se hace necesario plantearse los siguientes principios y valores que guíen los procesos educativos en las escuelas, las instituciones educativas y el quehacer diario de la sociedad: igualdad, solidaridad, la fidelidad a la palabra, el respeto al otro y la convivencia, el esfuerzo, la paz, el amor, el perdón y la información o el conocimiento frente a la ignorancia.

Las sociedades democráticas precisan de procesos educativos que vertebren sus políticas y acciones en los valores mencionados, sin olvidar que la igualdad, la solidaridad o la fraternidad favorecerán la consecución de las mismas. En palabras de Salazar \& Woldenberg (2016, p.43): "A fin de cuentas, aun si la democracia es esencialmente 
política, sólo la generalización de los valores democráticos en el conjunto de la sociedad permite un ejercicio pleno y funcional de la misma”. Es por ello, por lo que la escuela, su profesorado $y$ las familias, se pueden convertir en los mejores promotores de los principios democráticos.

La escuela: su compromiso en la educación en valores ante los retos de la Sociedad Red

Ante el planteamiento mostrado anteriormente, la actual "crisis de valores" y la ausencia de valores (Morín 1999; Bernardini, 2010; Mínguez, 2012; Piña, 2012; Berríos \& Buxarrais, 2013; Torrego \& Martínez, 2014; Arenas, 2015), en ocasiones de referentes universales, hace que la construcción de la personalidad de niños y jóvenes se vea mediatizada por el consumo de medios de comunicación, Internet o tecnologías. Ante este panorama, se hace preciso una educación en valores por parte de la escuela, buscando formas correlacionadas en las que se priorice la formación integral del niño, atendiendo a sus necesidades académicas, formativas y morales. Lo cierto, es que, en este sentido, los nińos se enfrentan continuamente a experiencias moralmente controvertidas, resolviendo conflictos de valor día a día, porque como individuos, los valores se van adquiriendo de forma progresiva desde los primeros ańos.

Educar en valores, más aún en la Sociedad Red, supone emplear la inteligencia moral de forma adecuada porque en las soluciones a conflictos individuales, cada persona pone en marcha sus competencias y habilidades, sus propias capacidades individuales y sus guías de valor (Martínez, Puig \& Trilla, 2003). Los valores morales que desde la escuela se desean desarrollar y proyectar en la ciudadanía se entienden en las prácticas diarias sirviendo de retroalimento de las guías de valor en los niños y niñas.

Actualmente, la familia y la escuela siguen siendo dos de las instituciones socializadoras más importantes, pero no se debe menospreciar la influencia que ejercen los medios de comunicación, Internet o las tecnologías en la vida de los niños y jóvenes, porque comparten la tarea de formación de estos (Martín \& Tyner, 2012; Cuervo, Medrano $\&$ Aierbe, 2016). La escuela, en este caso, se ve influenciada por la cultura de Internet, por los estilos de vida y comportamientos que aparecen en esta y por la formación en valores que invita a interiorizar, una nueva perspectiva que es importante cuidar, observar, reflexionar y tomar conciencia de los efectos que puede generar, porque la educación en valores y los procesos de alfabetización en todos los registros comunicativos permitirán a las personas ser críticas y responsables en la Sociedad Red que les ha tocado vivir (Ambrós \& Breu, 2007; García-Ruíz, Ramírez \& Rodríguez, 2014).

\section{Discusión y Conclusiones}

Una vez que se analizó toda la información documental de las diferentes bases de datos y el análisis de la literatura para la introducción y el apartado de revisión de la literatura, se llegó a las siguientes conclusiones, las cuales se enmarcan dentro de criterios de autores que aseveran la relevancia de los retos, oportunidades y ventajas de educar en valores hoy en día: una ética educativa comprometida con la escuela. Dentro de este contexto, hasta este momento se ha realizado un recorrido sobre Internet, los valores que precisa la sociedad actual y la finalidad de la educación en valores en este entramado. $Y$ es que el acceso solamente no garantiza un uso adecuado de Internet, ni una adecuada adquisición de valores porque:

Para que la Sociedad del Conocimiento sea una realidad y continúe contribuyendo al progreso mundial es necesario que los ciudadanos puedan acceder a las TIC de modo normalizado en las respectivas facetas de la vida. Reto que no solo está supeditado al propio conocimiento, sino que, además, se encuentra condicionado por las posibilidades de acceso y el uso que se hace de las herramientas digitales con toda la repercusión que esto conlleva para las personas (Rico, Sevillano \& Feliz, 2011, p.504).

Es, desde la reflexión, donde se exponen las siguientes pautas orientativas con la finalidad de arrojar luces a los nuevos procesos comunicativos a través de Internet, otorgando a los valores, su 
espacio para lograr una verdadera integración en la cotidianeidad de la escuela y en la búsqueda de una ciudadanía democrática, respetuosa y justa. Una sociedad desde la que se promuevan valores sociales y personales sustentados en la corresponsabilidad social, con implicación de todos los agentes educativos y buscando que niños y jóvenes encuentren a lo largo de su proceso formativo, las herramientas y orientaciones que les permitan ser libres, respetuosos y conscientes de sus acciones en la Red, (Figura 1).

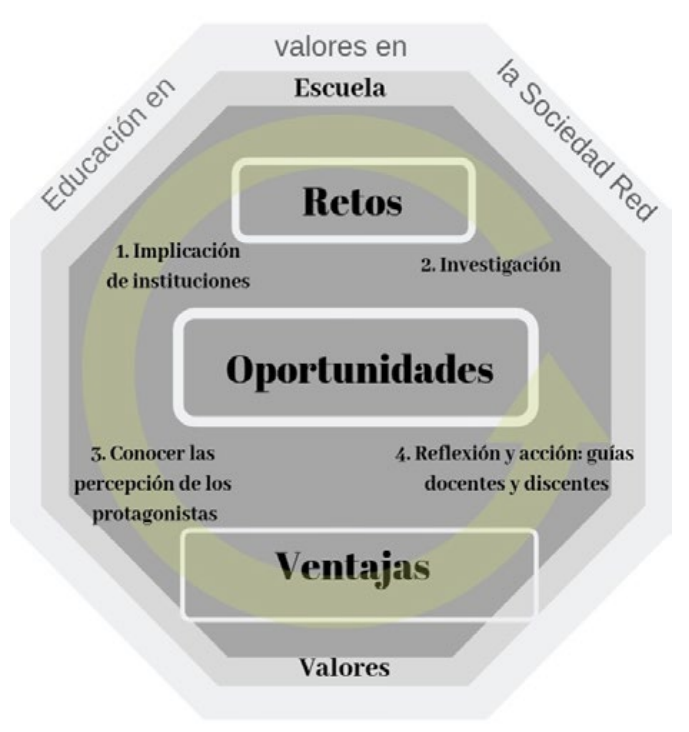

\section{Figura 1}

Retos, oportunidades y ventajas de la educación en valores en la Sociedad Red.

Fuente: Elaboración propia 2019

Otro aspecto relevante dentro de la promoción de valores es el trinomio escuela-familia-profesores, quienes deben promover el por, para y ante valores como principios democráticos, lo cual lo asevera también Reverter, 2004, p.109, al referir que la promoción de valores globales que exijan y construyan "modelos sociales y pautas de vida que respondan al mínimo establecido por una ética cívica transnacional”.

Dentro de este análisis se ha podido ver que sería interesante apelar a las instituciones políticas para que promuevan entre la ciudadanía una "ética cívica transnacional" (Cortina, 2003, p.133). Que cada país no solamente descubra por sí mismo los valores y principios que son compartidos por los ciudadanos, sino que ese diálogo y esa mirada se realice desde la ruptura de las fronteras, desde el inicio de los procesos transnacionales para que realmente se configure una ética cívica común y compartida. En palabras de Nussbaum (1999, p.51), "la educación es para las personas. Antes de poder elaborar un plan para el futuro de la educación, necesitamos entender los problemas que afrontamos en el proceso de transformación de los estudiantes en ciudadanos de la democracia, capaces de efectuar buenas reflexiones y elecciones sobre una gran variedad de temas de importancia nacional y mundial". Es decir, las instituciones políticas, sociales y educativas requieren plantearse el compromiso y la preservación de una ética cívica común y respetuosa con todas las personas en la sociedad global y tecnológica actual.

No se puede dejar de lado el rol importante que tienen las diversas instituciones públicas, sobre todo el invitar a controlar a las empresas para evitar la corrupción de tecnologías potencialmente peligrosas. Como diría Gee \& Esteban-Guitart (2019), algunas tecnologías permiten posibilidades de interacción, activismo y aprendizaje que en ocasiones se emplea inadecuadamente y es que el contexto en el que se utiliza la tecnología "tiene más peso en la hora de determinar sus efectos que la propia tecnología” (p.12).

Es importante también investigar sobre las percepciones de niños y jóvenes sobre los valores culturales y sociales que adquieren en su cotidianeidad a través del influjo de la Red, respetando su propia perspectiva (Pereira, 2013) y no la que se considera que deben tener (Livingstone \& Sefton-Green, 2016); buceando hacia el verdadero significado de las ideologías que suponen los efectos de las tecnologías y los medios (Erstad \& Sefton-Green, 2013; Pereira, S., Fillol \& Moura, 2019).

Desde una mirada más amplia, se hace necesario promover pautas educativas que tengan en cuenta la perspectiva de los diferentes agentes educativos, teniendo en cuenta sus miradas, percepciones y consideraciones, porque las tecnologías, Internet o los medios de comunicación cobran diferente sentido en función de la interpretación de cada individuo o grupo social (Hoffman, 2017; Yudes-Gómez, Baridon-Chauvie, \& González-Cabrera, 2018). 
Conocer de primera mano las verdaderas causas por las cuales los niños y jóvenes buscan en Internet o en las redes sociales el espacio para divertirse, relacionarse o comunicarse. No hay que olvidar que estos suelen buscar espacios online para establecer relaciones sociales (Ochaíta, Espinosa \& Gutiérrez, 2011) y, por tanto, como adultos tenemos el deber y la obligación moral de atender adecuadamente a estas necesidades y demandas, otorgándoles las herramientas suficientes que les permitan ser, estar y permanecer seguros, respetuosos y tolerantes en los espacios virtuales.

A futuro, hay que considerar favorecer iniciativas vinculadas a programas de formación infantojuvenil, así como a la elaboración de guías didácticas destinadas a profesionales de la educación en relación a cómo Internet o el mundo digitalizado en el que se enmarcan los niños y jóvenes con los que trabajan, influye en las relaciones inter e intrapersonales, en la adquisición de valores y en la construcción del juicio moral. La finalidad sería favorecer encuentros y espacios abiertos al diálogo, la participación y la escucha. Otro elemento sería la reflexión sobre la continua trasformación social y sus efectos en el desarrollo de la personalidad moral (Anaya, 2013, UNESCO, 2015) con la finalidad de promover la autonomía y la adquisición responsable de valores. Y promover el "aprendizaje profundo" (Gee, 2017), en el que se planteen objetivos claros de debate y diálogo sobre la influencia que ejerce algún contenido como aquellos que son objeto de análisis en este trabajo, que son los medios de comunicación, Internet o las tecnologías en la vida cotidiana, sus efectos en el desarrollo emocional y el descubrir cuáles son los valores y juicios de valor que intervienen en la toma de decisiones y en internacionalizar las normas.

\section{Agradecimientos}

Este trabajo se ha elaborado en el marco de Alfamed (Red Interuniversitaria Euroamericana de Investigación en Competencias Mediáticas para la Ciudadanía), con el apoyo del Proyecto $\mathrm{I}+\mathrm{D}+\mathrm{I}$ Coordinado "Competencias mediáticas de la ciudadanía en medios digitales emergentes (smartphones y tablets): prácticas innovadoras y estrategias educomunicativas en contextos múltiples" (EDU2015-64015-C3-1-R) (MINECO/ FEDER), y de la "Red de Educación Mediática" del Programa Estatal de Investigación Científica-Técnica de Excelencia, Subprograma Estatal de Generación de Conocimiento (EDU201681772-REDT), financiados por el Fondo Europeo de Desarrollo Regional (FEDER) y Ministerio de Economía y Competitividad de Espańa. Y también del Proyecto I+D de la Universidad de Cantabria "Competencia mediática en la sociedad digital para la participación crítica de la ciudadanía (UC2016-GRE-01)”.

\section{REFERENCIAS BIBLIOGRÁFICAS}

Abad, J.V. (2018). Promover la equidad en la escuela. Aula de innovación educativa, 275, 67.

Agarwal, R. \& Venkatesh, V. (2002). Assessing a firm`s web presence: A heuristic evaluation procedure for the measurement of usability. Information System Research, 13, 168225. https://doi.org/10.1287/isre.13.2.168.84

Agarwal, R. (2000). Individual acceptance of information technologies. In R. W. Zmud (Ed.), Framing the domains of it management research: glimpsing the future to the past. Pinnaflex, 85-104.

Albizuri I.E., Samaniego C.M. \& Torrientes, E.Q. (2001). Desarrollo de los valores en las instituciones educativas. Madrid: Mensajero.

Ambrós, A. \& Breu, R. (2007). Cine y educación. El cine en el aula de primaria y secundaria. Barcelona: Grao.

Anaya, R. (2013). El programa de Educación en Valores y su relación con el razonamiento moral, ideología educativa y liderazgo del Centro Docente. Nóesis: Revista de Ciencias Sociales y Humanidades, 22(44), 90-119. https://doi. org/10.20983/noesis.2013.3.4

Aparici, R (Coord.) (2010). Educomunicación: más allá del 2.0. Barcelona: Gedisa.

Arenas, A. (2015). Crisis del estado democrático: un acercamiento teórico y ético. Revista educación y desarrollo social, 10(1) 214-229.

Arribas, J. M. \& Torrego, J. C. (2008). Cómo elaborar y desarrollar el plan de convivencia. En J. C. Torrego (coord.) El plan de convivencia: Fundamentos y recursos para su elaboración y desarrollo (pp. 95-166). Madrid: Alianza Editorial.

Bauman, Z. (2007). Los retos de la educación en la modernidad líquida. Barcelona: Gedisa.

Bernardini, A. (2010). La educación en valores hoy en día: entre conciencia crítica y respuestas constructivas. Innovaciones Educativas, 17, 11-22. 
Berríos, Ll. \& Buxarrais Estrada, M. R. (2013). Educación en valores: análisis sobre las expectativas y los valores de los adolescentes. Educación y educadores, 16(2), 244-264. https://doi.org/10.5294/edu.2013.16.2.3

Besalú, X. (2002). Diversidad cultural y educación. Madrid: Síntesis Educación.

Bindé, J. (2004). Introdução geral. In J. Bindé (Ed.), Para onde vão os valores? Lisboa: Instituto Piaget.

Briñol, P., Horcajo, J., De la Corte, L., Valle, C., Gallardo, I. \& Díaz, D. (2004). Efecto de la ambivalencia evaluativa sobre el cambio de actitudes. Psicothema. 16, 373-377

Buxarrais, M., Martínez, M., Puig, J. \& Trilla, J. (1990). La educación moral en primaria y secundaria. Madrid: Edelvives.

Buxarrais, R.M. (1997). La formación del profesorado en educación en valores. Bilbao: Desclée de Brouwer.

Cabrera, D. (2004). La matriz imaginaria de las nuevas tecnologías. Comunicación y Sociedad, 17 (1), 9-45.

Caldeiro, MC. \& Aguaded, J. (2015). «Estoy aprendiendo, no me molestes» la competencia mediática como forma de expresión crítica de nativos e inmigrantes digitales. Redes. com, 12. Recuperado de http://goo.gl/2ORkf1

Carmel, E. \& Agarwal, R. (2002). The maturation of offshore sourcing of information technology work. MIS Quarterly Executive, 1, 65-77

Castells, M. (2003). La era de la información. Economía, sociedad y cultura: 2. El poder de la identidad. Madrid: Alianza.

Colom, T., \& Melich, J. C. (1993). Postmodernidad y educación. La teoría de Toffler y la práctica de la C. M. U. Teoría de la Educación. Revista Interuniversitaria, 5, 97-110.

Cortina, A. (2003). Ética Cívica Transnacional: Fundamentación y Aplicación. Seminarios de filosofía, 16, 119-134. Recuperado de https://goo.gl/FtLkki

Cuervo, S. L., Medrano, C. \& Aierbe, A. (2016). Values perceived by adolescents on television: cross-cultural and gender differences. Educación XX1, 19(2), 383-404.

Day, R. A. (2005). Cómo escribir y publicar trabajos científicos ( $3^{\mathrm{a}}$ ed.). Washington, DC: Organización Panamericana de Salud.

Deval J., 2002. La escuela posible. Barcelona: Ariel.

Erstad, O., \& Sefton-Green, J. (2013). Digital disconect? The 'digital learner' and the school. In O. Erstad, \& J. Sefton-Green (Eds.), Identity, community, and learning lives in the digital age (pp. 87-104). New York: Cambridge University Press. https://doi.org/10.3726/978-1-4539-1019-1

Fernández, S., \& López, C. (2018). La sociedad en red y el cambio de nuestra percepción. Hologramatica, 28 (1), 135153.

García Aretio, L., Ruíz, M., \& García Blanco, M. (2010). Claves para la Educación. Madrid: Narcea.
García-Ruíz, R., Ramírez, A. \& Rodríguez, M. M. (2014). Media Literacy Education for a New Prosumer Citizenship. Comunicar, 22, (43), 15 - 23.https://doi.org/10.3916/C432014-01

García-Ruiz, R., Ramírez-García, A \& Rodríguez, M.M. (2014). Educación en alfabetización mediática para una nueva ciudadanía prosumidora. Comunicar, 43, 15-23. https://doi.org/10.3916/C43-2014-01

Garitaonandia \& Garmendia (2007). Cómo usan Internet los jóvenes: hábitos, riesgos y control parental [Proyecto Eukids online]. Vizcaya: Universidad del País Vasco, Facultad de Ciencias Sociales y de la Comunicación [Documento en línea]. Recuperado de http://www.anobium.es/docs/gc_fichas/doc/01237BEXqx.pdf

Garrido-Cabezas, N. (2011). Internet como un nuevo medio social para la comunicación y la participación ciudadana. Revista de comunicación Vivat Academia, 117E, 13751390. https://doi.org/10.15178/va.2011.117E.1375-1390

Gee, J.P. (2017). Teaching, learning, literacy in our high-risk high-tech world: A framework for becoming human. New York: Teachers College Press.

Hernández, J.M. \& Eyeang, E. (2017). Los valores en la educación de África. De ayer a hoy. Salamanca: Ediciones Universidad de Salamanca.

Hernández-Serrano, M., Renés-Arellano, P., Graham, G. \& Greenhill, A. (2017). From Prosumer to Prodesigner: Participatory News Consumption. Comunicar, 50, 77-88. https://doi.org/10.3916/C50-2017-07

Hoffmann, A.L. (2017). Breaking bad algorithms. Science, 358, 310-311. https://doi.org/10.1126/science.aao4414

Jiménez, J. (2002). La invención del arte. El término Techné. Teoría del arte; Tecnos-Alianza, Madrid.

Koh, C. (2014). Exploring the use of Web 2.0 technology to promote moral and psychosocial development: Can YouTube work? British Journal of Educational Technology, 45 (4), pp. 619-635. https://doi.org/10.1111/bjet.12071

Livingstone, S. \& Sefton-Green, J. (2016). The class: living and learning in the digital age. New York: New York University Press. https://doi.org/10.18574/ nyu/9781479884575.001.0001

Maceiras, M. (2015). Una aproximación filosófica. La herencia de la filosofía clásica y su vigencia en la actualidad. Revista del consejo escolar del estado, 4(6), 7-17. https:// doi.org/10.4438/1886-5o97-PE

Maeda, J. (2006). Laws of Simplicity. U.S.A: M.I.T Press.

Martín, A. G. \& Tyner, K. (2012). Media Literacy in Multiple Contexts. Comunicar, 19 (38), 10-12. https://doi. org/10.3916/C38-2012-02-00

Martínez, J. M. (2015). La participación de los padres en la educación. Un análisis crítico. Participación educativa, 4(7), 65-70.

Martínez, M., Esteban, F., \& Buxarrais, M.R. (2011). Escuela, profesorado y valores, Revista de educación, N. ${ }^{\circ}$ Extra. $1,95-113$. 
Martínez, M., Puig, J. M. \& Trilla, J. (2003). Escuela, profesorado y educación moral. Teoría de la educación, 15, 57-94.

Mayor, J. (2007). La educación en valores, una apuesta de la sociedad. Idea La Mancha. Revista de Educación de Castilla-La Mancha, 271-274. Recuperado de http://redined.mecd.gob.es/xmlui/bitstream/ handle/11162/93584/00620073000950.pdf?sequence=1

Medina, A \& Ballano, S. (2015). Retos y problemáticas de la introducción de la educación mediática en los centros de secundaria. Revista de Educación, 369, 135-158. https://doi. org/10.4438/1988-592\#-RE-2015-369-293.

Mínguez, R. (2012). La responsabilidad educativa en tiempo de crisis. Edetania, 42, 107-125.

Mínguez, R. (2014). Ética de la vida familiar y transmisión de valores morales. Revista de educación, 363, 2010-229.

Morín, E. (1999). Los siete saberes necesarios a la educación del futuro. París: UNESCO.

Nussbaum, M. (1999). Sin fines de lucro. Por qué la democracia necesita de las humanidades. Madrid: Katz Ediciones.

Ochaíta, E., Espinosa, M.A. \& Gutiérrez, H. (2011). Las necesidades adolescentes y las nuevas tecnologías de la información y la comunicación. Revista de Estudios de Juventud, 92, 87-110.

ONU, Asamblea General, Declaración Universal de los Derechos Humanos. Recuperado de http://www.un.org/es/ universal-declaration-human-rights/

Orantes, S. (2011). Viabilidad del Modelo de la Aceptación de la Tecnología en las empresas mexicanas. Una aproximación a las actitudes y percepciones de los usuarios de las tecnologías de la información. Revista Digital Universitaria, $12,1-15$.

Ortega, P. \& Minguez, R. (2004). familia y transmisión de valores. Teoría de la Educación. Revista interuniversitaria, 15, 33-56. https://goo.gl/y762Fp

Pereira, S. (2013). More Technology, Better Childhoods? The Case of the Portuguese 'One Laptop per Child' Programme. CM: Communication Management Quarterly, 29, 171-198. https://doi.org/10.5937/comman1329171P

Pereira, S., Fillol, J. \& Moura, P. (2019). Young people learning from digital media outside of school: The informal meets the formal. [El aprendizaje de los jóvenes con medios digitales fuera de la escuela: De lo informal a lo formal]. Comunicar, 58, 41-50. https://doi.org/10.3916/C58-2019-04

Piña, E. (2012). Aprender en el vivir. Revista educare, 16, 2, 138-158.

Reig, D. (2018). Jóvenes de un nuevo mundo: cambios cognitivos, sociales, en valores, de la Generación conectada. Revista de estudios de juventud, 108, 21-32. Recuperado de https://goo.gl/nmueBQ

Reverter, J. (2004). Valores básicos de la identidad europea. Castellón de la Plana: Universitas.

Rico, MC., Sevillano, ML. \& Feliz, T. (2011). Competencias necesarias para la utilización de las principa- les herramientas de Internet en la educación. Revista de educación, 356, 483-507. https://doi.org/10-4438/1988592X-RE-2010-356-048

Ruiz, M., Bernal, A., Gil, F., \& Escámez Sánchez, J. (2012). Ser uno mismo. Repensando la autonomía y la responsabilidad como coordenadas de la educación actual. Teoría de la Educación. Revista Interuniversitaria, 24(2), 59-81.

Salazar, L. \& Woldenberg, J. (2016). Principios y valores de la democracia. Cuadernos de Divulgación de la Cultura Democrática, Instituto Nacional Electoral: México. Recuperado de http://biblio.ine.mx/janium/Documentos/cuaderno_01.pdf

Tornero, J.M. (2017). Aprender a ser con Internet. Barcelona: Octaedro.

Torrego, J. C., \& Martínez, C. (2014). Claves para el Desarrollo del Plan de Convivencia en los Centros Educativos desde una Perspectiva Integral. Qualitative Research in Education, 3 (1), 83-113.

UNESCO. (2015). Replantear la educación. ¿Hacia un bien común mundial? Recuperado de http://unesdoc.unesco. org/images/0023/002326/232697s.pdf

Yudes-Gómez, C., Baridon-Chauvie, D. \& González-Cabrera, J.M. (2018). Cyberbylling and problematic Internet use in Colombia, Uruguay and Spain: Cross-cultural study. [Ciberacoso y uso problemático de Internet en Colombia, Uruguay y España: Un estudio transcultural]. Comunicar, 56, 49-58. https://doi.org/10.3916/C56-2018-05

Zhao, S., Grasmuck, S. \& Martin, J. (2008). Identity construction on Facebook: Digital empowerment in anchored relationships. Computers in Human Behavior, 24, 18161836. https://doi.org/10.1016/j.chb.2008.02.012 